\title{
Application of Cationic Conjugated Polymer-Outer Membrane Vesicle Complexes in Inhibiting Red Blood Cell Aggregation
}

\author{
Ping $\mathrm{He}^{\mathrm{a}}$ \\ Endong Zhang a,b \\ Ruilian $\mathbf{Q i}^{\mathbf{a}}$ \\ Fengting $\mathbf{L v}^{\mathrm{a}}$ \\ Libing Liu*a,b (iD) \\ Shu Wang ${ }^{\mathrm{a}, \mathrm{b}}$ (i) \\ ${ }^{a}$ Beijing National Laboratory for Molecular Sciences, Institute of Chemistry, \\ Chinese Academy of Sciences, Beijing, China \\ ${ }^{\mathrm{b}}$ School of Chemistry and Chemical Engineering, \\ University of Chinese Academy of Sciences, Beijing, China \\ liulibing@iccas.ac.cn
}

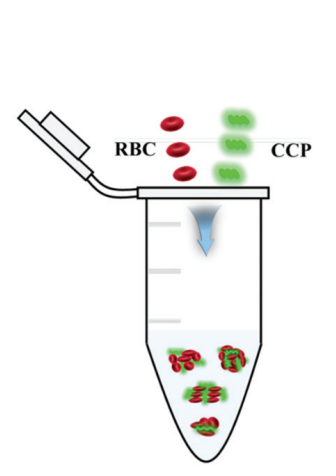

RBC aggregation

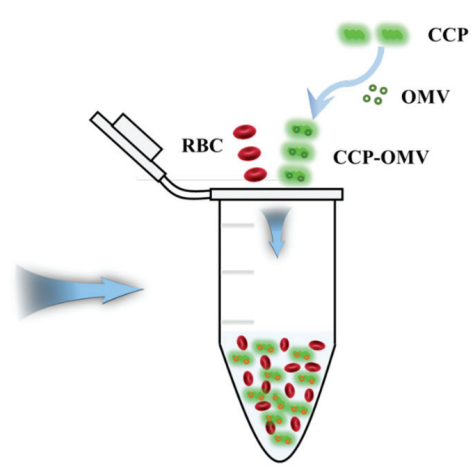

Inhibited RBC aggregation
Received: 06.08.2019

Accepted after revision: 16.09.2019

DOI: 10.1055/s-0039-1700849; Art ID: om-19-0007-rc

License terms: CCO

(c) 2019. The Author(s). This is an open access article published by Thieme under the terms of the Creative Commons Attribution-NonDerivative-NonCommercial-License, permitting copying and reproduction so long as the original work is given appropriate credit. Contents may not be used for commercial purposes, or adapted, remixed, transformed or built upon. (https://creativecommons.org/licenses/by-nc-nd/4.0/).

Abstract Cationic conjugated polymers (CCPs) have been attracting a lot of attention in biomedical applications because of their good photoelectric properties and good cell viability. However, positively charged components may reduce the electrostatic repulsion among red blood cells (RBCs) and induce RBC aggregation, which may lead to thrombus. Herein, in this work, we prepared the complexes of CCPs and outer membrane vesicles (OMVs) to inhibit RBC aggregation induced by CCPs and improve the biocompatibility of CCPs. A poly(fluorene-co-phenylene) derivative (PFP), a poly(p-phenylenevinylene) derivative (PPV), and a poly(thiophene) derivative (PMNT) were chosen as the model CCPs, and OMVs were used as the representative of a cell membrane. The CCP-OMV complexes were formed mainly driven by electrostatic interactions. Besides, the electrostatic CCP-OMV complexes were proved to be able to prevent the RBC aggregation induced by CCPs while maintaining the hydrophobic interactions between CCPs and RBCs.

Key words cationic conjugated polymers, outer membrane vesicles, inhibition of RBC aggregation

\section{Introduction}

Red blood cells (RBCs) are the most abundant cells in blood and take responsibility for transporting oxygen and carbon dioxide. ${ }^{1}$ Generally, the electrostatic repulsion which comes from the surficial negative charge of RBCs maintains the suspension stability of RBCs. However, the increase of positively charged proteins in blood will lead proteins to absorbing onto the surface of RBCs and neutralize the negative charge, reducing the electrostatic repulsion among RBCs and inducing RBC aggregation. Then the RBC aggregation may cause thrombus and threaten human life and health. ${ }^{2}$

Conjugated polymers ( $\mathrm{CPs}$ ) have been attracting much attention in the applications of biosensing and imaging, ${ }^{3}$ bacterial detection and inhibition, ${ }^{4}$ and cancer diagnosis and therapy, ${ }^{5}$ due to their good photoelectric properties, such as light harvesting, signal amplification, high quantum yield, and good photostability. The introduction of positively charged groups can not only improve the water solubility of CPs, but also facilitate the interactions between CPs and negatively charged biological components (e.g. DNA, ${ }^{6}$ cells, ${ }^{7}$ bacteria ${ }^{8}$ ) through electrostatic attraction. Nevertheless, the nonspecific electrostatic attraction may cause unwished influences to other negatively charged components while interacting with targets, for instance, the induction of RBC aggregation. Thus, improving the biocompatibility of cationic CPs (CCPs) is of great importance in propelling the biomedical applications of CPs.

Recently, cell membrane-coated nanoparticles have received a lot of attention and have seen developments in biomedical fields. This membrane-coating technology can enhance the efficiency of materials by extending blood circulation time and inducing immune response. ${ }^{9}$ Inspired by this technology, we used outer membrane vesicles (OMVs) of Pseudomonas aeruginosa (P. aeruginosa) as a cell membrane model and used a poly(fluorene-co-phenylene) derivative (PFP), ${ }^{10}$ a poly(p-phenylenevinylene) derivative (PPV), ${ }^{11}$ and a poly(thiophene) derivative (PMNT) ${ }^{12}$ as typical CCPs, to form CCP-OMV complexes. CCPs displayed relatively good biocompatibility, for instance, PMNT could even promote cell growth without serum. We discovered that CCP-OMVs can effectively avoid the RBC aggregation induced by CCPs, which proved the improved biocompatibility of CCP by forming CCP-OMV complexes. 


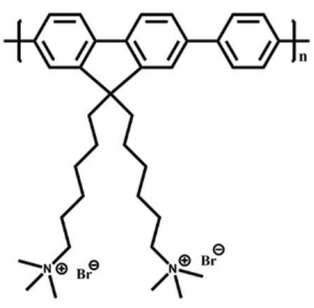

PFP

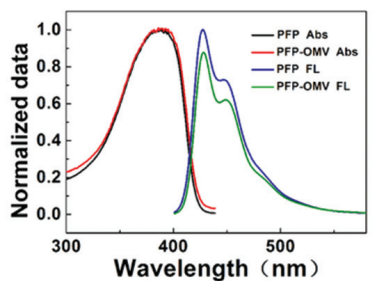

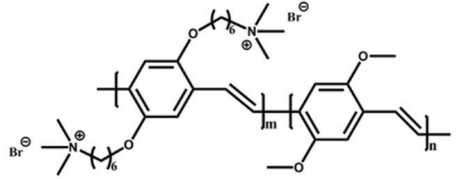

PPV

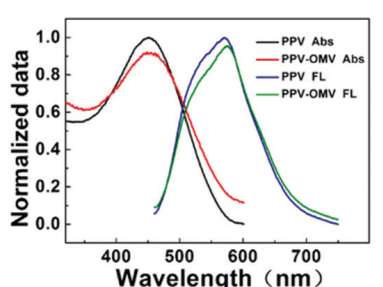

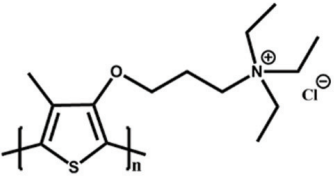

PMNT

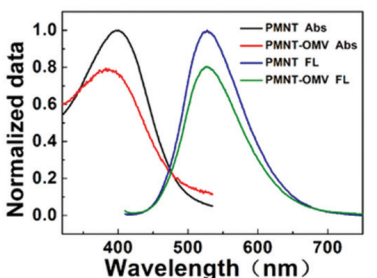

Fig. 1 The UV-Vis and fluorescence spectra of CCPs before and after the formation of CCP-OMV complexes.

\section{Results and Discussion}

The morphology of OMVs was characterized by scanning electron microscopy (SEM) and transmission electron microscopy (TEM). The zeta potential and size of OMVs were measured to be $-18.8 \pm 3.2 \mathrm{mV}$ and $120 \mathrm{~nm}$, respectively (see Figure $S 1$ in the Supporting Information).

The UV-Vis absorption and fluorescence emission spectra of CCPs in water were measured before and after the complex formed (Figure 1). CCPs used in this work were PFP, PPV, and PMNT, whose $\lambda_{\text {max,abs }} / \lambda_{\text {max,em }}$ values are $393 /$ $427,451 / 570$, and $410 / 527 \mathrm{~nm}$, respectively. After the formation of the electrostatic CCP-OMV complexes, the spectral properties of PFP and PPV were scarcely influenced. While for PMNT, both of the absorption and emission spectrum intensities decreased and the maximum absorption wavelength had a slight blue-shift from 401 to $387 \mathrm{~nm}$, which might result from conformational change of PMNT in the complex aggregate.

The CCP-OMV complexes were formed by mixing OMV solution $(80 \mu \mathrm{g} / \mathrm{mL})$ and CCP solution at room temperature for $4 \mathrm{~h}$. The detailed procedure of preparation of CCP-OMVs is given in the References and Notes section..$^{13}$ And the CCPOMV complexes were characterized through laser scanning confocal microscopy (LSCM; Figure 2), TEM (see Figure S2 in the Supporting Information), and SEM (see Figure S3 in the Supporting Information), which indicated the formation of CCP-OMV complexes. As shown in Figure 2, the interactions between CCPs and OMVs would strengthen along with the increase of CCP concentration. Besides, PFP displayed the

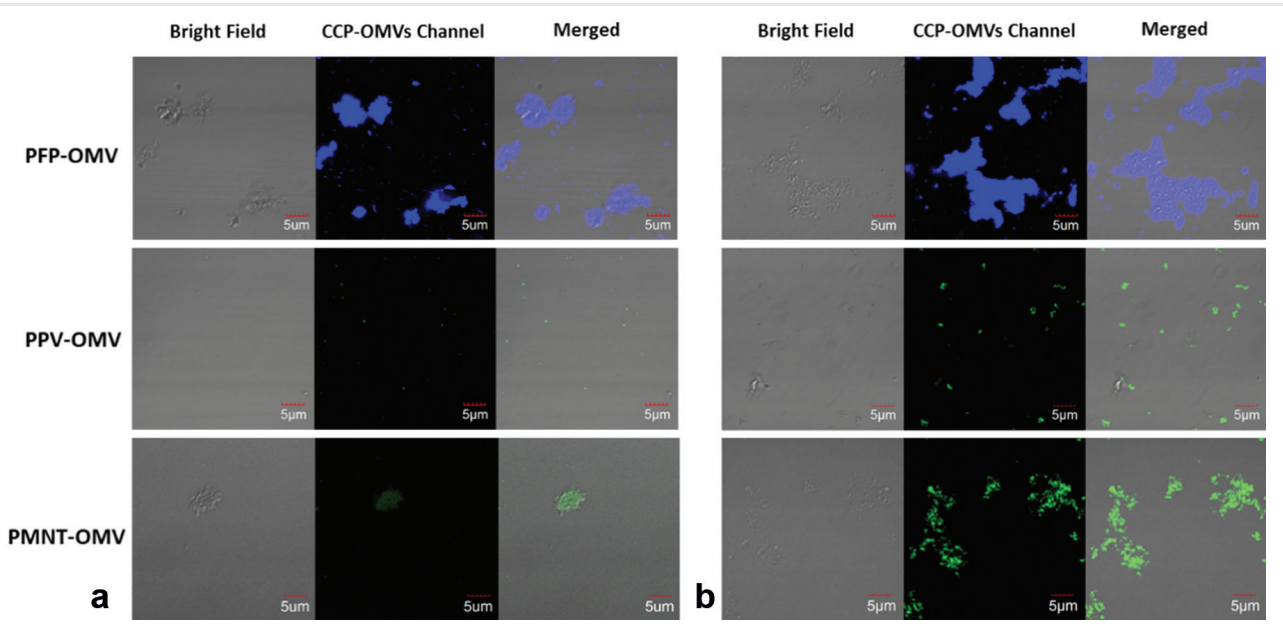

Fig. 2 LSCM characterization of the formation of CCP-OMV complexes. (a) [CCP] $=10 \mu \mathrm{mol} / \mathrm{L}$; (b) [CCP] $=100 \mu \mathrm{mol} / \mathrm{L}$. 
strongest interaction with OMVs among these three CCPs under the same conditions.

To have a good knowledge of the interaction process between CCPs and OMVs, the zeta potential of every component was measured. The thermodynamic change during the formation of CCP-OMV complexes was also measured. As shown in Figure 3a, the zeta potential values of PFP, PPV, and PMNT were $20.8 \pm 3.9,26.1 \pm 1.7$, and $16.0 \pm 5.9 \mathrm{mV}$, respectively. After forming complexes with OMVs $(-18.8 \pm 3.2 \mathrm{mV})$, the zeta potential values of PFPOMV, PPV-OMV, and PMNT-OMV complexes were $21.7 \pm 0.9,-2.7 \pm 0.2,-2.9 \pm 0.2 \mathrm{mV}$, respectively. Compared with the corresponding CCPs, the zeta potential of PPV-OMVs and PMNT-OMVs showed certain negative movements and finally presented weak electronegativity. While for PFP, it seemed that the zeta potential did not change before and after the complex formed. Considering the LSCM results, it was inferred that the strong interaction between PFP and OMVs made OMVs wrapped in PFP, finally presenting the zeta potential of PFP. For the enthalpy change $\Delta \boldsymbol{H}_{\text {obs }}$ shown in Figure $3 \mathrm{~b}$, with the addition of PPV solution into OMVs, the $\Delta \boldsymbol{H}_{\mathrm{obs}}$ changed from a large negative value to zero, which indicated a gradually saturated exothermal process. The zeta potential and enthalpy change suggested that the electrostatic interaction made the most contributions for the formation of CCP-OMV complexes.

Then we investigated the interactions between CCPs or CCP-OMVs and RBCs under different concentrations (10 and $100 \mu \mathrm{mol} / \mathrm{L}$; Figure 4). For PFP, it could cause RBC aggregation at $10 \mu \mathrm{mol} / \mathrm{L}$ instead of $100 \mu \mathrm{mol} / \mathrm{L}$, which was attributed to the self-aggregation of PFP in physiological saline solution at $100 \mu \mathrm{mol} / \mathrm{L}$. While for PPV, it could lead to RBC aggregation at both 10 and $100 \mu \mathrm{mol} / \mathrm{L}$. Furthermore, the aggregation extent strengthened with the increase of PPV concentration. PMNT induced RBC aggregation at $100 \mu \mathrm{mol} / \mathrm{L}$ other than at $10 \mu \mathrm{mol} /$ $\mathrm{L}$, which indicated a concentration-dependent aggregation induction way. The above three CCPs showed different RBC aggregation induction effects because of their structural characteristics and properties. Unignorably, all of them are potential inducers of RBC aggregation when applied in a biomedical field.

To verify the effects of CCP-OMVs in inhibiting RBC aggregation induced by CCPs, the interactions between CCP-OMVs and RBCs were also investigated under the same conditions. PPV-OMVs and PMNT-OMVs did not induce RBC aggregation at neither 10 nor $100 \mu \mathrm{mol} / \mathrm{L}$, displaying an effective inhibition of RBC aggregation. PFP-OMVs avoided $\mathrm{RBC}$ aggregation at $10 \mu \mathrm{mol} / \mathrm{L}$, but induced RBC aggregation at $100 \mu \mathrm{mol} / \mathrm{L}$. In combination with the zeta potential of CCP-OMVs, it was deduced that electronegative PPV-OMVs and PMNT-OMVs were not able to neutralize the surficial positive charge of RBCs and thus could not induce RBC aggregation. However, the electropositive PFP-OMVs might act as a cationic center which absorbed surrounding RBCs and induced aggregation.

Likewise, to have a better understanding of the interaction mechanisms between CCPs and CCP-OMVs with RBCs, zeta potentials were measured and the thermodynamic changes during the interaction of PPV and PPV-OMVs with RBCs were also measured. As shown in Figure 5a, the zeta potential of $\mathrm{RBCs}$ in physiological saline solution was $-12.9 \pm 1.5 \mathrm{mV}$. After interacting with PFP and PFP-OMVs, the zeta potential values changed to $-11.9 \pm 1.1$ and $-8.99 \pm 0.7 \mathrm{mV}$, respectively, suggesting a stronger electrostatic interaction between PFP-OMVs and RBCs than that with PFP. In contrast, the zeta potential values of RBCs changed to $-8.83 \pm 0.9$ and -11.0 $\pm 0.6 \mathrm{mV}$ after interacting with PPV and PPV-OMVs, suggesting a stronger electrostatic attraction between PPV and RBCs than that with PPV-OMVs. The results are consistent with the LSCM results (Figure 2). For PMNT and PMNT-OMVs, both of them did not cause obvious changes in RBC zeta potential, which might be attributed to the relatively low electric charge density of PMNT and weaker electrostatic interaction between PMNT with RBCs.

As shown in Figure 5b, the interactions of RBCs with PPV and PPV-OMVs both were endothermal processes, indicating
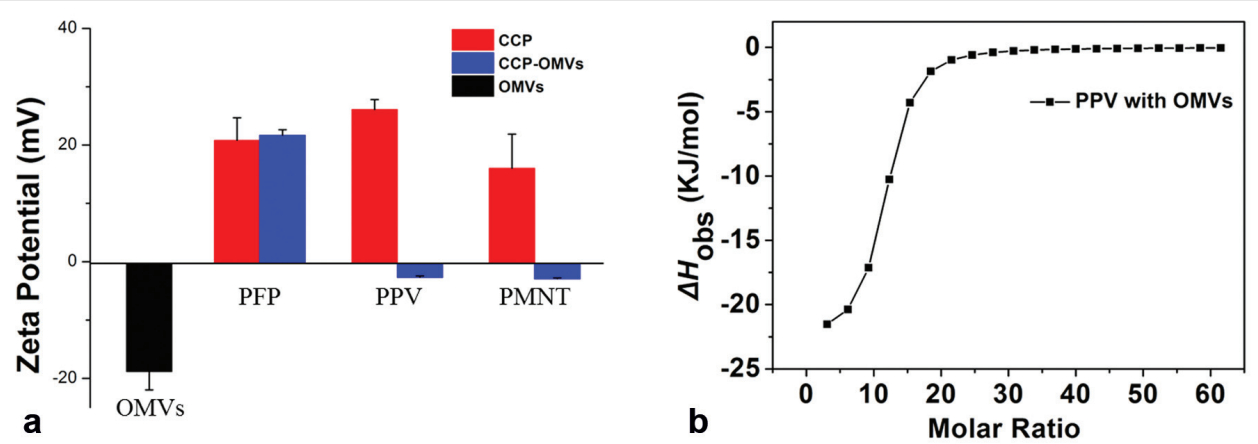

Fig. 3 (a) The zeta potential of OMVs, CCPs, and corresponding complexes. (b) The enthalpy changes $\left(\Delta \boldsymbol{H}_{\text {obs}}\right)$ against the PPV/OMV molar ratio by titrating the aqueous solution of $240 \mu \mathrm{mol} / \mathrm{L}$ PPV into OMV solution. The dilution enthalpy of PPV has been deducted. 


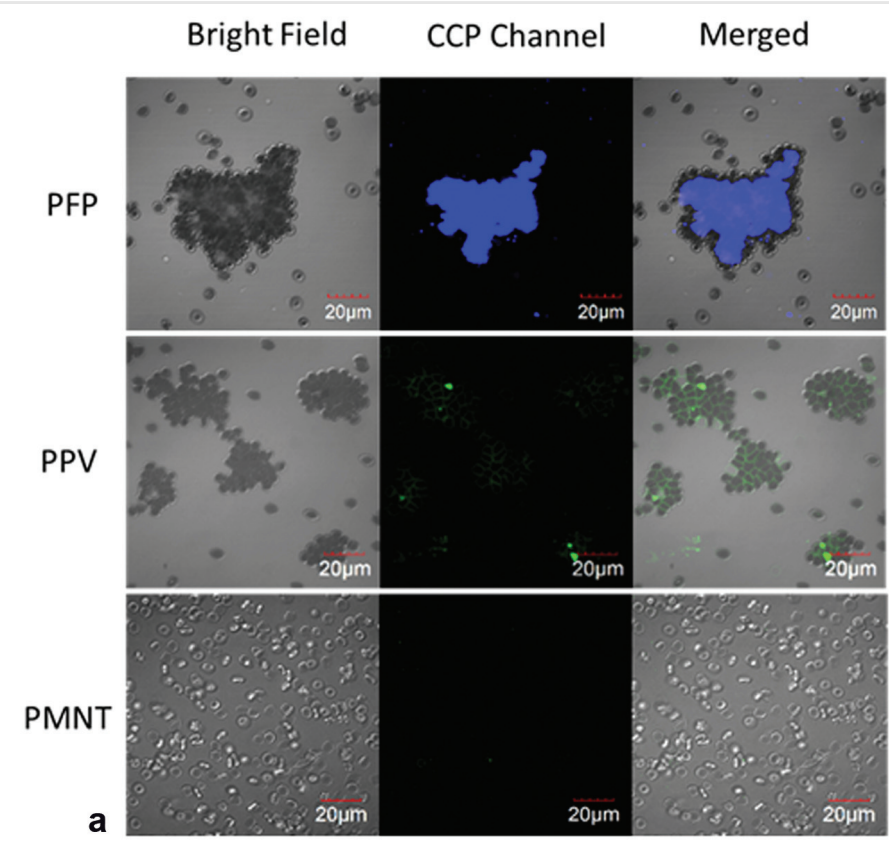

Bright Field CCP-OMV Channel Merged

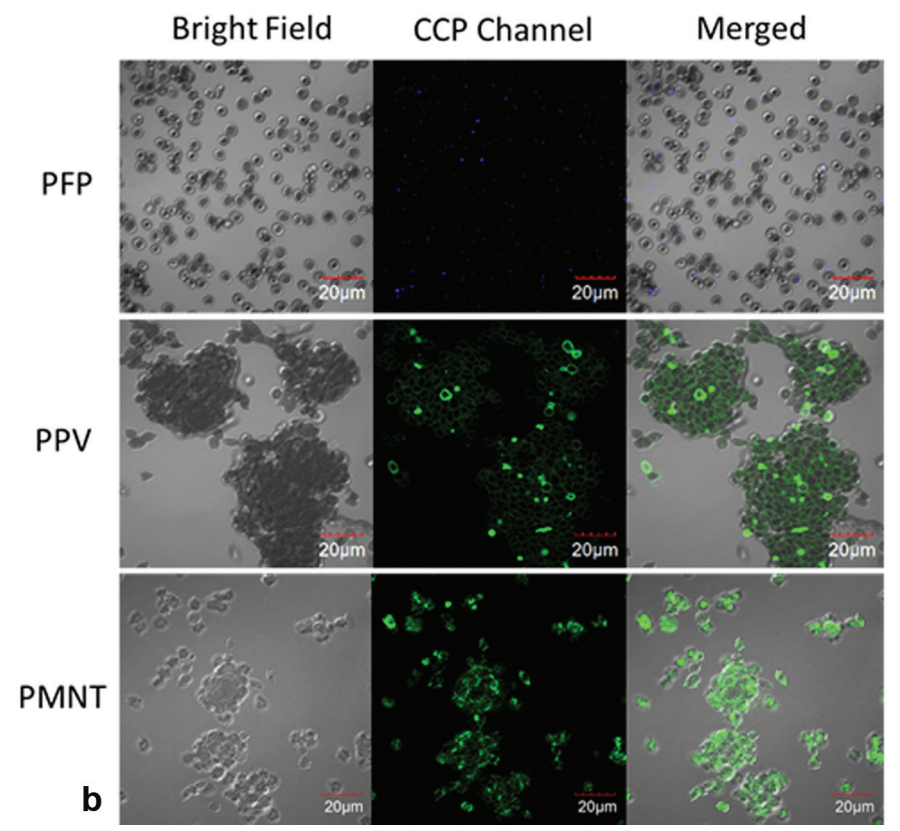

Fig. 4 Microscopic characterization of RBC aggregation induced by CCPs and CCP-OMVs. (a) $10 \mu \mathrm{mol} / \mathrm{L}$. (b) $100 \mu \mathrm{mol} / \mathrm{L}$.

the main driving force of the hydrophobic interaction. Besides, learning from the binding constant $\left(K_{a}\right)$ and binding ratio $(N)$ (see Table S1 in the Supporting Information), PPVOMVs displayed stronger interaction than PPV. Therefore, the formation of PPV-OMV complexes not only avoided inducing RBC aggregation, but also retained the hydrophobic interaction between PPV and RBCs.
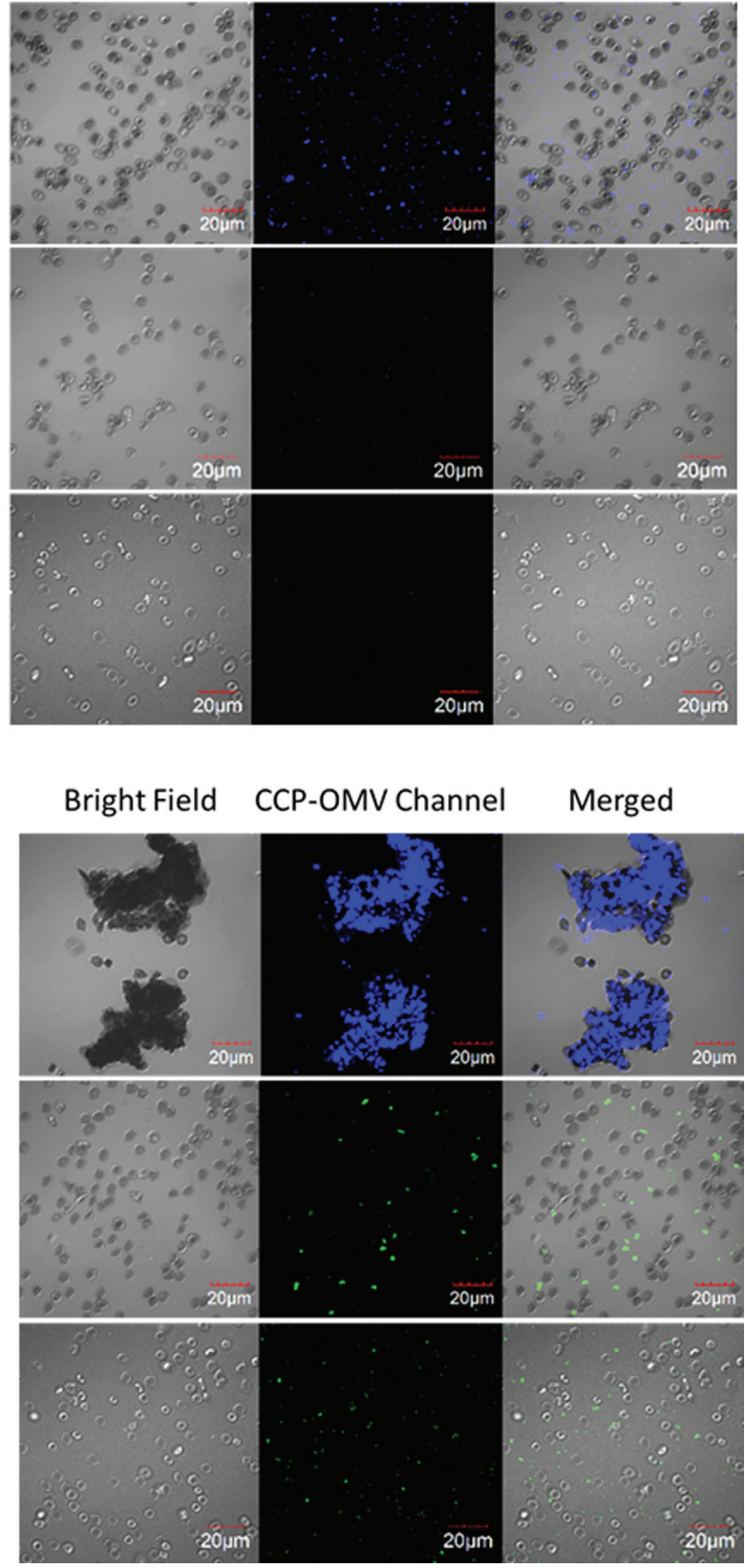

Bright Field CCP-OMV Channel Merged

\section{Conclusion}

In summary, we used OMVs of $P$. aeruginosa as the cell membrane model to form electrostatic complexes with CCPs. The complexes were proved to be able to inhibit the RBC aggregation induced by CCPs, which was demonstrated by LSCM. The zeta potential and enthalpy change during the 

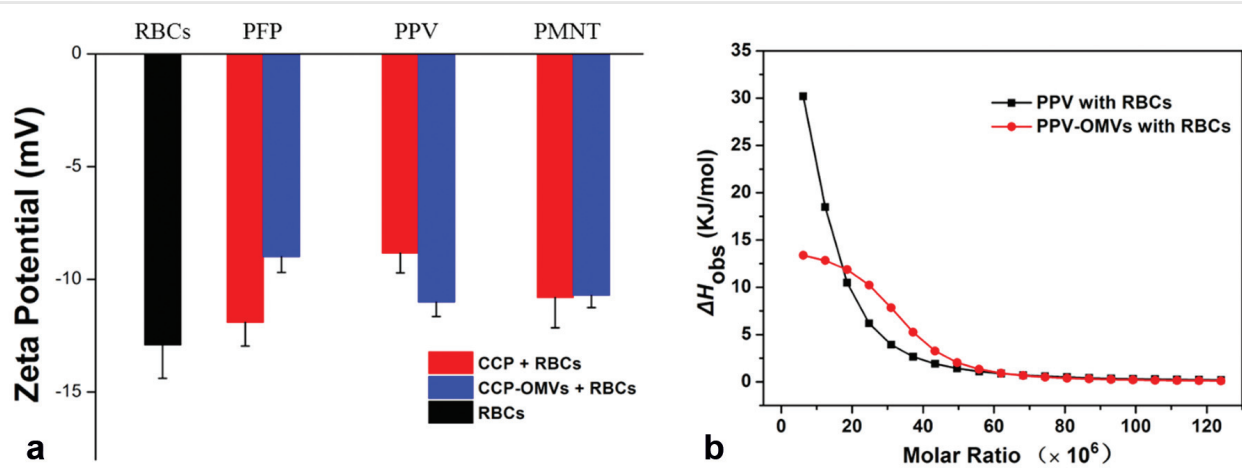

Fig. 5 (a) The zeta potentials of RBCs before and after interacting with CCPs and CCP-OMVs. (b) The enthalpy changes ( $\triangle \boldsymbol{H}_{\mathrm{obs}}$ ) against the PPV/RBCs and PPV-OMV/RBC molar ratio by titrating the physiological saline solution of $240 \mu \mathrm{mol} / \mathrm{L}$ PPV or PPV-OMVs into RBCs solution, respectively. The dilution enthalpies of PPV and PPV-OMVs have been deducted.

formation of complexes indicated the electrostatic force actuation between CCP and OMVs, which effectively reduced the impact on the superficial electronegativity of RBCs. Thus, CCP-OMVs were able to avoid inducing RBC aggregation. Besides, the zeta potential and enthalpy change during the interaction of CCPs and CCP-OMVs with RBCs also suggested the non-negligible function of electrostatic and hydrophobic interactions in this process. In brief, the CCP-OMV complexes were proved to be prospective in inhibiting the RBC aggregation induced by CCPs while retaining the interactions with RBCs.

\section{Funding Information}

The authors would like to thank the National Natural Science Foundation of China (21773268, 21533012, and 21661132006) for financial support.

\section{Acknowledgement}

The authors appreciate Prof. Yilin Wang at Key Laboratory of Colloid, Interface, and Chemical Thermodynamics, Institute of Chemistry, Chinese Academy of Sciences for ITC measurements.

\section{Supporting Information}

Supporting information for this article is available online at https://doi.org/10.1055/s-0039-1700849.

\section{References and Notes}

(1) Wang, T. T.; Jiang, X. ACS Appl. Mater. Interfaces 2015, 7, 129.

(2) (a) Han, Y.; Wang, X.; Dai, H.; Li, S. ACS Appl. Mater. Interfaces 2012, 4, 4616. (b) Jan, K. M.; Chien, S. J. Gen. Physiol. 1973, 61, 638. (c) Jan, K. M. Bioelectrochemistry 1980, 188, 143. (d) Snabre,
P.; Grossmann, G. H.; Mills, P. Colloid Polym. Sci. 1985, 263, 478. (e) Armstrong, J. K.; Meiselman, H. J.; Fisher, T. C. Am. J. Hematol. 1997, 56, 26. (f) Toth, K.; Wenby, R. B.; Meiselman, H. J. Biorheology 2000, 37, 301. (g) Byrnes, J. R.; Wolberg, A. S. Blood 2017, 130, 1795.

(3) Zhu, C. L.; Liu, L. B.; Yang, Q.; Lv, F. T.; Wang, S. Chem. Rev. 2012, $112,4687$.

(4) (a) Disney, M. D.; Zheng, J.; Swager, T. M.; Seeberger, P. H. J. Am. Chem. Soc. 2004, 126, 13343. (b) Yuan, H. X.; Liu, Z.; Liu, L. B.; Lv, F. T.; Wang, Y. L.; Wang, S. Adv. Mater. 2014, 26, 4333. (c) Zhu, C. L.; Yang, Q.; Liu, L. B., et al. Adv. Mater. 2011, 23, 4805.

(5) (a) Li, M.; Li, S. L.; Chen, H.; Hu, R.; Liu, L. B.; Lv, F. T.; Wang, S. ACS Appl. Mater. Interfaces 2016, 8, 42. (b) Li, M.; He, P.; Li, S. L., et al. ACS Biomater. Sci. Eng. 2018, 4, 2037.

(6) (a) Duan, X. R.; Li, Z. P.; He, F.; Wang, S. J. Am. Chem. Soc. 2007, 129, 4154. (b) Xia, F.; Zuo, X. L.; Yang, R. Q., et al. J. Am. Chem. Soc. 2010, 132, 1252.

(7) (a) Zhu, C. L.; Yang, Q.; Liu, L. B.; Wang, S. Chem. Commun.(Camb.) 2011, 47, 5524. (b) Wang, B.; Nie, C. Y.; Liu, L. B.; Lv, F. T.; Wang, S. Chin. Chem. Lett. 2017, 28, 1975.

(8) (a) Feng, G. G.; Mai, C. K.; Zhan, R. Y.; Bazan, G. C.; Liu, B. J. Mater. Chem. B Mater. Biol. Med. 2015, 3, 7340. (b) Huang, Y.; Pappas, H. C.; Zhang, L. Q., et al. Chem. Mater. 2017, 29, 6389.

(9) (a) Dehaini, D.; Fang, R. H.; Zhang, L. F. Bioeng. Transl. Med. 2016, 1, 30. (b) Kroll, A. V.; Fang, R. H.; Zhang, L. F. Bioconjug. Chem. 2017, 28, 23. (c) Su, J. H.; Sun, H. P.; Meng, Q. S., et al. Adv. Funct. Mater. 2016, 26, 1243. (d) Gao, W. W.; Fang, R. H.; Thamphiwatana, S., et al. Nano Lett. 2015, 15, 1403. (e) Hu, C. MJ.; Fang, R. H.; Copp, J.; Luk, B. T.; Zhang, L. F. Nat. Nanotechnol. 2013, 8, 336. (f) Yang, G.; Liu, L.; Lv, F.; Wang, S. Sci. Rep. 2013, 3, 1702.

(10) Gaylord, B. S.; Massie, M. R.; Feinstein, S. C.; Bazan, G. C. Proc. Natl. Acad. Sci. U.S.A. 2005, 102, 34.

(11) Yuan, H. X.; Liu, Z.; Liu, L. B.; Lv, F. T.; Wang, Y. L.; Wang, S. Adv. Mater. 2014, 26, 4333-4338.

(12) Liu, X. F.; Tang, Y. L.; Wang, L. H.; Zhang, J.; Song, S. P.; Fan, C. H.; Wang, S. Adv. Mater. 2007, 19, 1471-1474.

(13) The preparation of CCP-OMVs complex: PPV storage solution (10 $\mathrm{mM}$ ) was added into the OMVs solution $(80 \mu \mathrm{g} / \mathrm{mL})$ to obtain a final concentration of PPV in $10 \mu \mathrm{M}$ or $100 \mu \mathrm{M}$. Then the mixture was placed in room temperature for $4 \mathrm{~h}$ to obtain PPV-OMVs. PFP-OMVs and PMNT-OMVs were prepared by the same way as PPV-OMVs. 